\title{
On Minimal Model Theory for Algebraic Log Surfaces
}

\author{
Osamu Fujino
}

\begin{abstract}
We introduce the notion of generalized MR log canonical surfaces and establish the minimal model theory for generalized MR log canonical surfaces in full generality.
\end{abstract}

\section{Introduction}

In this short paper, we give some remarks on the minimal model theory for log surfaces defined over an algebraically closed field $k$ of any characteristic. We recall the definition of log surfaces following 3 .

Definition 1.1 (Log surfaces). Let $X$ be a normal algebraic surface and let $\Delta$ be an effective $\mathbb{R}$-divisor whose coefficients are less than or equal to one such that $K_{X}+\Delta$ is $\mathbb{R}$ Cartier. Then $(X, \Delta)$ is called a log surface. If $(X, \Delta)$ is a log surface and $X$ is $\mathbb{Q}$-factorial, then we simply say that $(X, \Delta)$ is a $\mathbb{Q}$-factorial log surface.

In 3, 9] (see also [7), Osamu Fujino and Hiromu Tanaka established the minimal model theory for $\mathbb{Q}$-factorial log surfaces in full generality. We note that a $\mathbb{Q}$-factorial log surface is not necessarily log canonical.

Definition 1.2 (Log canonical surfaces). Let $(X, \Delta)$ be a log surface and let $f: Y \rightarrow X$ be a proper birational morphism from a normal surface $Y$. In this situation, we can write

$$
K_{Y}+\Delta_{Y}=f^{*}\left(K_{X}+\Delta\right)
$$

with $f_{*} \Delta_{Y}=\Delta$. If the coefficients of $\Delta_{Y}$ are less than or equal to one for every $f: Y \rightarrow X$, then $(X, \Delta)$ is called a log canonical surface.

We note that a $\log$ canonical surface is not always $\mathbb{Q}$-factorial. Therefore, we can not directly apply the minimal model theory for $\mathbb{Q}$-factorial log surfaces to log canonical surfaces. Fortunately, however, we know that the minimal model theory works in full generality for log canonical surfaces by [3, 9].

Received April 22, 2020; Accepted January 11, 2021.

Communicated by Yoichi Miyaoka.

2020 Mathematics Subject Classification. Primary: 14E30; Secondary: 14J17.

Key words and phrases. log surfaces, log canonical surfaces, MR log canonical surfaces, generalized MR log canonical surfaces, minimal model program, abundance theorem. 
Let $\varphi: X \rightarrow W$ be a $\left(K_{X}+\Delta\right)$-negative extremal birational contraction morphism from a $\log$ canonical surface $(X, \Delta)$. Then the exceptional locus of $\varphi$ is disjoint from non-rational singular points of $X$ (see [4, Section 4]). This is a key point of the minimal model theory for (not necessarily $\mathbb{Q}$-factorial) log canonical surfaces.

In [1], Valery Alexeev introduced the notion of $M R \log$ canonical surfaces, which is a slight generalization of that of log canonical surfaces. Then, in [1, Section 10], he sketched the minimal model theory for MR log canonical surfaces.

Definition 1.3 (MR $\log$ canonical surfaces). Let $(X, \Delta)$ be a $\log$ surface and let $f: Y \rightarrow X$ be the unique minimal resolution of singularities of $X$. Then we can write

$$
K_{Y}+\Delta_{Y}=f^{*}\left(K_{X}+\Delta\right)
$$

with $f_{*} \Delta_{Y}=\Delta$. If the coefficients of $\Delta_{Y}$ are less than or equal to one, then we say that $(X, \Delta)$ is an $M R$ log canonical ( $M R L C$, for short) surface.

We note that $\Delta_{Y}$ in Definition 1.3 is always effective by the negativity lemma since $f: Y \rightarrow X$ is the minimal resolution of singularities of $X$. By definition, a log canonical surface is obviously an MR log canonical surface. However, an easy example (see Example 3.3 shows that there exists an MR log canonical surface which is not log canonical. Therefore, the minimal model theory established in [3, 9] (see also [7]) does not cover the minimal model theory for MR log canonical surfaces sketched in [1, Section 10]. In this paper, we introduce the following new notion of generalized MR log canonical surfaces.

Definition 1.4 (Generalized MR log canonical surfaces). Let $(X, \Delta)$ be a log surface. If there exists a proper birational morphism $f: Y \rightarrow X$ from a $\mathbb{Q}$-factorial normal surface $Y$ such that $\Delta_{Y}$ is an effective $\mathbb{R}$-divisor on $Y$ and that the coefficients of $\Delta_{Y}$ are less than or equal to one, where

$$
K_{Y}+\Delta_{Y}=f^{*}\left(K_{X}+\Delta\right)
$$

with $f_{*} \Delta_{Y}=\Delta$, then we say that $(X, \Delta)$ is a generalized $M R \log$ canonical (GMRLC, for short) surface.

By definition, $\mathbb{Q}$-factorial log surfaces, log canonical surfaces, and MR log canonical surfaces are all generalized MR log canonical surfaces. Then we establish the minimal model theory for generalized MR log canonical surfaces as follows.

Theorem 1.5 (Minimal model program for generalized MR log canonical surfaces). Let $(X, \Delta)$ be a generalized $M R$ log canonical surface and let $\pi: X \rightarrow S$ be a projective morphism onto a variety $S$. Then we can run the minimal model program over $S$ with respect 
to $K_{X}+\Delta$ by the cone and contraction theorem. Hence there exists a sequence of at most $\rho(X / S)-1$ birational contractions starting from $(X, \Delta)$

$$
(X, \Delta)=:\left(X_{0}, \Delta_{0}\right) \stackrel{\varphi_{0}}{\longrightarrow}\left(X_{1}, \Delta_{1}\right) \stackrel{\varphi_{1}}{\longrightarrow} \cdots \stackrel{\varphi_{l-1}}{\longrightarrow}\left(X_{l}, \Delta_{l}\right)=:\left(X^{*}, \Delta^{*}\right)
$$

over $S$ such that $\left(X_{i}, \Delta_{i}\right)$, where $\Delta_{i}:=\varphi_{i-1 *} \Delta_{i-1}$, is generalized MR log canonical for every $i$ and that one of the followings holds.

(i) (Good minimal model). If $K_{X}+\Delta$ is pseudo-effective over $S$, then $K_{X^{*}}+\Delta^{*}$ is semi-ample over $S$.

(ii) (Mori fiber space). If $K_{X}+\Delta$ is not pseudo-effective over $S$, then there is a contraction morphism $g: X^{*} \rightarrow W$ over $S$ onto a normal variety $W$, which is projective over $S$, with connected fibers such that $-\left(K_{X^{*}}+\Delta^{*}\right)$ is $g$-ample, $\operatorname{dim} W<2$, and the relative Picard number $\rho\left(X^{*} / W\right)$ is one.

Moreover, we have the following properties.

(1) If $(X, \Delta)$ is log canonical, then so is $\left(X_{i}, \Delta_{i}\right)$ for every $i$.

(2) If $X$ is $\mathbb{Q}$-factorial, then so is $X_{i}$ for every $i$.

(3) If $(X, \Delta)$ is MR log canonical, then so is $\left(X_{i}, \Delta_{i}\right)$ for every $i$.

More precisely, we prove the cone and contraction theorem for log surfaces in full generality (see Theorem 5.5). Let $\varphi: X \rightarrow Z$ be a $\left(K_{X}+\Delta\right)$-negative extremal birational contraction. Then we can check that if $(X, \Delta)$ is generalized MR log canonical then so is $\left(Z, \varphi_{*} \Delta\right)$ (see Lemma 4.3). Therefore, we can freely run the minimal model program for generalized MR log canonical surfaces. Hence we establish the minimal model theory for generalized MR log canonical surfaces. We note that Theorem 1.5 contains the abundance theorem for generalized MR log canonical surfaces, which is highly nontrivial and is new.

We will treat algebraic surfaces defined over any algebraically closed field $k$ of arbitrary characteristic throughout this paper. We will freely use the standard notation and the results in [2, 3, 9, (see also [5,7]).

\section{Preliminaries}

In this section, we collect some basic definitions.

Definition 2.1 (Operations for $\mathbb{R}$-divisors). Let $D=\sum_{i} d_{i} D_{i}$ be an $\mathbb{R}$-divisor on a normal surface such that $D_{i}$ is a prime divisor and $d_{i}$ is a real number for every $i$ and that $D_{i} \neq D_{j}$ for $i \neq j$. Then $\lceil D\rceil$ (resp. $\lfloor D\rfloor$ ) denotes the round-up (resp. round-down) of $D$. We put $\{D\}:=D-\lfloor D\rfloor$ and call it the fractional part of $D$. 
Definition 2.2 ( $\mathbb{R}$-linear equivalence). Let $\Delta_{1}$ and $\Delta_{2}$ be $\mathbb{R}$-Cartier divisors on a normal surface. Then $\Delta_{1} \sim_{\mathbb{R}} \Delta_{2}$ means that $\Delta_{1}$ is $\mathbb{R}$-linearly equivalent to $\Delta_{2}$.

We recall the definitions of klt surfaces, log canonical surfaces, and multiplier ideal sheaves.

Definition 2.3 (KLT surfaces, log canonical surfaces, and multiplier ideal sheaves). Let $(X, \Delta)$ be a $\log$ surface as in Definition 1.1 and let $f: Y \rightarrow X$ be a proper birational morphism from a normal surface $Y$. Then we can write

$$
K_{Y}+\Delta_{Y}=f^{*}\left(K_{X}+\Delta\right)
$$

with $f_{*} \Delta_{Y}=\Delta$. If the coefficients of $\Delta_{Y}$ are less than or equal to one (resp. less than one) for every $f: Y \rightarrow X$, then $(X, \Delta)$ is called a log canonical surface (resp. klt surface).

We further assume that $Y$ is smooth and $\operatorname{Supp} \Delta_{Y}$ is a simple normal crossing divisor on $Y$. Then we put

$$
\mathcal{J}(X, \Delta):=f_{*} \mathcal{O}_{Y}\left(-\left\lfloor\Delta_{Y}\right\rfloor\right)=f_{*} \mathcal{O}_{Y}\left(\left\lceil K_{Y}-f^{*}\left(K_{X}+\Delta\right)\right\rceil\right)
$$

and call it the multiplier ideal sheaf of the pair $(X, \Delta)$. It is well known that $\mathcal{J}(X, \Delta)$ is independent of the resolution $f: Y \rightarrow X$. We note that $\mathcal{J}(X, \Delta) \subset \mathcal{O}_{X}$ holds, that is, $\mathcal{J}(X, \Delta)$ is an ideal sheaf on $X$. We can check that $(X, \Delta)$ is klt if and only if $\mathcal{J}(X, \Delta)=$ $\mathcal{O}_{X}$ holds.

Let us recall a useful vanishing theorem for log surfaces.

Theorem 2.4 (Kawamata-Viehweg-Nadel vanishing theorem for log surfaces). Let $(X, \Delta)$ be a $\log$ surface and let $\pi: X \rightarrow S$ be a proper surjective morphism onto a variety $S$. When the characteristic of the base field $k$ is positive, we further assume that $\operatorname{dim} S \geq 1$. Let $L$ be a Cartier divisor on $X$ such that $L-\left(K_{X}+\Delta\right)$ is $\pi$-nef and $\pi$-big. Then

$$
R^{i} \pi_{*}\left(\mathcal{O}_{X}(L) \otimes \mathcal{J}(X, \Delta)\right)=0
$$

holds for every $i>0$, where $\mathcal{J}(X, \Delta)$ is the multiplier ideal sheaf of $(X, \Delta)$.

Sketch of proof. If the characteristic of $k$ is zero, then we can assume that $k=\mathbb{C}$ by the Lefschetz principle. In this case, the statement is well known (see [5, Theorem 3.4.2]). If the characteristic of $k$ is positive, then the desired vanishing theorem follows from 10 , Theorem 2.7] (see also [11, Section 3]).

We close this section with the following well-known result on projectivity.

Lemma 2.5. Let $X$ be a normal $\mathbb{Q}$-factorial surface. Then $X$ is always quasi-projective. Proof. The proof of [3, Lemma 2.2] works as well over any algebraically closed field $k$ of arbitrary characteristic. 
3. MR log canonical surfaces and generalized MR log canonical surfaces In this section, we explain various definitions and some examples. The following lemmas are obvious by definition. We state them explicitly for the reader's convenience.

Lemma 3.1. Let $(X, \Delta)$ be a log canonical surface. Then $(X, \Delta)$ is MR log canonical. Proof. Let $f: Y \rightarrow X$ be the minimal resolution of singularities of $X$. Then we can write

$$
K_{Y}+\Delta_{Y}=f^{*}\left(K_{X}+\Delta\right)
$$

as usual. By the definition of log canonical surfaces (see Definition 2.3), the coefficients of $\Delta_{Y}$ are less than or equal to one. This means that $(X, \Delta)$ is MR log canonical.

Lemma 3.2. Let $(X, \Delta)$ be a log surface such that $X$ is smooth. Then $(X, \Delta)$ is $M R \log$ canonical.

Proof. Since $X$ is smooth, $X$ itself is the minimal resolution of singularities of $X$. Therefore, $(X, \Delta)$ is MR log canonical because the coefficients of $\Delta$ are less than or equal to one by the definition of log surfaces (see Definition 1.1).

We note that an MR log canonical surface is not necessarily log canonical.

Example 3.3. We put $X=\mathbb{P}^{2}$ and $\Delta=L_{1}+L_{2}+L_{3}$, where $L_{1}, L_{2}$, and $L_{3}$ are three distinct lines passing through a point $P$. Then $(X, \Delta)$ is MR log canonical by Lemma 3.2 . On the other hand, we can check that $(X, \Delta)$ is not log canonical by taking a blow-up of $X$ at $P$.

We summarize the relationship between various definitions.

Lemma 3.4. We have the following properties.

(i) If $(X, \Delta)$ is MR log canonical, then it is automatically generalized MR log canonical.

(ii) If $(X, \Delta)$ is a $\mathbb{Q}$-factorial log surface, then it is generalized $M R$ log canonical.

(iii) A log canonical surface is not necessarily $\mathbb{Q}$-factorial.

(iv) $A \mathbb{Q}$-factorial log surface is not always log canonical.

(v) $A \mathbb{Q}$-factorial log surface is not always $M R \log$ canonical.

Proof. By definition, (i) is obvious because smooth surface is automatically $\mathbb{Q}$-factorial. Let $(X, \Delta)$ be a $\mathbb{Q}$-factorial log surface. Then the identity map of $X$ satisfies the condition of Definition 1.4. Therefore, $(X, \Delta)$ is generalized MR log canonical. This is (ii). It is well 
known that a log canonical surface is not always $\mathbb{Q}$-factorial by the classification of $\log$ canonical surface singularities. The simplest example is the cone over an elliptic curve. Hence (iii) holds. We note that surfaces with only rational singularities are automatically $\mathbb{Q}$-factorial. On the other hand, two-dimensional rational singularities are not necessarily $\log$ canonical. Therefore, we obtain (iv). We can easily construct a projective toric surface $X$ with only one singular point $P$ of type $A_{1}$. It is well known that $X$ is $\mathbb{Q}$-factorial. Let $D_{1}$ and $D_{2}$ be two general Cartier divisors on $X$ passing through $P$. Then $(X, \Delta)$, where $\Delta=D_{1}+D_{2}$, is a $\mathbb{Q}$-factorial log surface. However, $(X, \Delta)$ is not MR log canonical at $P$. Hence we have (v). Of course, (iv) is a special case of $(\mathrm{v})$.

By (ii) and (v) in Lemma 3.4, a generalized MR log canonical surface is not always MR log canonical. Moreover, there exists a generalized MR log canonical surface which is not $\mathbb{Q}$-factorial by Lemmas 3.1 and 3.4 (iii).

\section{Basic properties of generalized MR log canonical surfaces}

Let us start with the abundance theorem for GMRLC surfaces. It easily follows from 3 , Theorem 8.1] and [9, Theorem 6.7].

Theorem 4.1 (Abundance theorem for GMRLC surfaces). Let $(X, \Delta)$ be a GMRLC surface and let $\pi: X \rightarrow S$ be a proper surjective morphism onto a variety $S$. Assume that $K_{X}+\Delta$ is $\pi$-nef. Then $K_{X}+\Delta$ is $\pi$-semi-ample.

Proof. By definition, there exist a proper birational morphism $f: Y \rightarrow X$ such that $K_{Y}+\Delta_{Y}=f^{*}\left(K_{X}+\Delta\right)$ and that $\left(Y, \Delta_{Y}\right)$ is a $\mathbb{Q}$-factorial log surface. It is obvious that $K_{Y}+\Delta_{Y}$ is $\pi \circ f$-nef. Therefore, $K_{Y}+\Delta_{Y}$ is $\pi \circ f$-semi-ample by the abundance theorem for $\mathbb{Q}$-factorial $\log$ surfaces (see [3, Theorem 8.1] and [9, Theorem 6.7]). Thus, we obtain that $K_{X}+\Delta$ is $\pi$-semi-ample.

As an easy consequence of Theorem 4.1, we have a weak version of the basepoint-free theorem for GMRLC surfaces. This weak version seems to be almost sufficient for many applications. We will treat more general results in Theorems 5.1 and 5.4 .

Corollary 4.2. Let $(X, \Delta)$ be a GMRLC surface and let $\pi: X \rightarrow S$ be a projective morphism onto a variety $S$. Let $D$ be a $\pi$-nef Cartier divisor on $X$ such that $D-\left(K_{X}+\Delta\right)$ is $\pi$-ample. Then $D$ is $\pi$-semi-ample.

Proof. Without loss of generality, we may assume that $S$ is affine and that $\pi_{*} \mathcal{O}_{X} \simeq \mathcal{O}_{S}$ holds. By Bertini's theorem, we can write $D \sim_{\mathbb{R}} K_{X}+\Delta+A+\pi^{*} B$ for some ample $\mathbb{R}$-divisor $A$ on $X$ and an $\mathbb{R}$-Cartier divisor $B$ on $S$ such that $(X, \Delta+A)$ is GMRLC. By Theorem 4.1, $K_{X}+\Delta+A$ is $\pi$-semi-ample since it is $\pi$-nef. Hence $D$ is $\pi$-semi-ample. 
The following lemma is a key result for GMRLC surfaces.

Lemma 4.3. Let $(X, \Delta)$ be a GMRLC (resp. an MRLC) surface and let $\varphi: X \rightarrow Z$ be a proper birational morphism onto a normal surface $Z$ such that $-\left(K_{X}+\Delta\right)$ is $\varphi$-nef. Then $\left(Z, \Delta_{Z}\right)$ is GMRLC (resp. MRLC), where $\Delta_{Z}=\varphi_{*} \Delta$.

Proof. First, we assume that $(X, \Delta)$ is GMRLC. We take a proper birational morphism $f: Y \rightarrow X$ from a $\mathbb{Q}$-factorial normal surface $Y$ with $K_{Y}+\Delta_{Y}=f^{*}\left(K_{X}+\Delta\right)$ as in Definition 1.4. Since $-\left(K_{X}+\Delta\right)$ is $\varphi$-nef, $-\left(K_{Y}+\Delta_{Y}\right)$ is nef over $Z$. Then, by the negativity lemma, we can uniquely take an $\mathbb{R}$-divisor $\Theta$ on $Y$ with $\Theta \leq \Delta_{Y}$ such that $-\left(K_{Y}+\Theta\right)$ is numerically trivial over $Z$ and $(\varphi \circ f)_{*} \Theta=\Delta_{Z}$. We write $\Theta=\Theta_{+}-\Theta_{-}$, where $\Theta_{+}$and $\Theta_{-}$are both effective $\mathbb{R}$-divisors with no common components. By construction, $K_{Y}+\Theta_{+}$is numerically equivalent to $\Theta_{-}$over $Z$ and $\Theta_{-}$is exceptional over $Z$. We note that $Y$ is projective over $Z$ since it is $\mathbb{Q}$-factorial (see Lemma 2.5). Thus we can run the minimal model program over $Z$ with respect to $K_{Y}+\Theta_{+}$(see [3, Theorem 3.3] and $\left[9\right.$, Theorem 6.5]). Then we finally get a $\mathbb{Q}$-factorial $\log$ surface $\left(Y^{*}, \Theta_{+}^{*}\right)$ such that $K_{Y^{*}}+\Theta_{+}^{*}$ is numerically trivial over $Z$. By the abundance theorem (see [3, Theorem 8.1] and $\left[9\right.$, Theorem 6.7]), $K_{Y^{*}}+\Theta_{+}^{*}$ is $\mathbb{R}$-linearly equivalent to zero over $Z$. By construction, $p_{*}\left(K_{Y^{*}}+\Theta_{+}^{*}\right)=K_{Z}+\Delta_{Z}$ holds, where $p: Y^{*} \rightarrow Z$, and $K_{Z}+\Delta_{Z}$ is $\mathbb{R}$-Cartier. Thus, we have $K_{Y^{*}}+\Theta_{+}^{*}=p^{*}\left(K_{Z}+\Delta_{Z}\right)$. This means that $\left(Z, \Delta_{Z}\right)$ is GMRLC because $\left(Y^{*}, \Theta_{+}^{*}\right)$ is a $\mathbb{Q}$-factorial log surface.

Next, we assume that $(X, \Delta)$ is MRLC. Then we may assume that $f: Y \rightarrow X$ is the minimal resolution of singularities of $X$ in the above argument. Then we can easily see that $Y^{*}$ is a smooth surface. More precisely, $Y \rightarrow Y^{*}$ is a composition of contractions of $(-1)$-curves. If there exists a $(-1)$-curve in the fiber of $p: Y^{*} \rightarrow Z$, then we contract it to a smooth point. By repeating this process finitely many times, we may further assume that $p: Y^{*} \rightarrow Z$ is the minimal resolution of singularities of $Z$. Therefore, $\left(Z, \Delta_{Z}\right)$ is MRLC.

As an easy application of Lemma 4.3 , we prove the following useful lemma although we do not use it explicitly in this paper.

Lemma 4.4. Let $(X, \Delta)$ be a GMRLC (resp. an $M R L C)$ surface and let $\Delta^{\prime}$ be an effective $\mathbb{R}$-divisor on $X$ such that $\Delta^{\prime} \leq \Delta$. Then $\left(X, \Delta^{\prime}\right)$ is GMRLC (resp. MRLC).

Proof. We take a proper birational morphism $f: Y \rightarrow X$ from a $\mathbb{Q}$-factorial normal surface $Y$ with $K_{Y}+\Delta_{Y}=f^{*}\left(K_{X}+\Delta\right)$ as in Definition 1.4. We note that $f_{*}^{-1}\left(\Delta-\Delta^{\prime}\right)$ is $f$ nef. Therefore, we obtain that $-\left(K_{Y}+\Delta_{Y}-f_{*}^{-1}\left(\Delta-\Delta^{\prime}\right)\right)$ is $f$-nef. We also note that $f_{*}\left(\Delta_{Y}-f_{*}^{-1}\left(\Delta-\Delta^{\prime}\right)\right)=\Delta^{\prime}$ by construction. Of course, $\left(Y, \Delta_{Y}-f_{*}^{-1}\left(\Delta-\Delta^{\prime}\right)\right)$ is GMRLC since $Y$ is $\mathbb{Q}$-factorial. Therefore, by Lemma 4.3. $\left(X, \Delta^{\prime}\right)$ is GMRLC. When 
$(X, \Delta)$ is MRLC, we can assume that $Y$ is smooth in the above argument. In this case, $\left(Y, \Delta_{Y}-f_{*}^{-1}\left(\Delta-\Delta^{\prime}\right)\right)$ is MRLC. Therefore, $(X, \Delta)$ is MRLC by Lemma 4.3 .

5. Minimal model theory for generalized MR log canonical surfaces

In this section, we discuss the minimal model theory for GMRLC surfaces. Let us start with the basepoint-free theorem for log surfaces (see also [11, Theorem 4.2]).

Theorem 5.1 (Basepoint-freeness for $\log$ surfaces I). Let $(X, \Delta)$ be a log surface such that $\lfloor\Delta\rfloor=0$ and let $\pi: X \rightarrow S$ be a projective surjective morphism onto a variety $S$. Let $D$ be a $\pi$-nef Cartier divisor on $X$ such that aD $-\left(K_{X}+\Delta\right)$ is $\pi$-nef and $\pi$-big for some positive integer a. Then there exists a positive integer $m_{0}$ such that $\mathcal{O}_{X}(m D)$ is $\pi$-generated for every integer $m \geq m_{0}$.

Before we explain the proof of Theorem 5.1, we prepare two easy non-vanishing theorems.

Lemma 5.2 (Non-vanishing theorem I). Let $C$ be a smooth projective curve and let $G$ be a $\mathbb{Q}$-divisor on $C$ such that $\lfloor G\rfloor \leq 0$. Let $D$ be a nef Cartier divisor on $C$ such that $a D-\left(K_{C}+G\right)$ is ample for some positive integer a. Then $H^{0}\left(C, \mathcal{O}_{C}(m D+\lceil-G\rceil)\right) \neq 0$ for every integer $m \geq a$.

Proof. If $C \simeq \mathbb{P}^{1}$, then $H^{0}\left(C, \mathcal{O}_{C}(m D+\lceil-G\rceil)\right) \neq 0$ for every non-negative integer $m$ since $\operatorname{deg} D \geq 0$ and $\lceil-G\rceil \geq 0$. If $C \not \mathbb{P}^{1}$, then we can easily check that $H^{0}\left(C, \mathcal{O}_{C}(m D+\right.$ $\lceil-G\rceil)) \neq 0$ for every $m \geq a$ by the Riemann-Roch formula. More precisely, we have

$$
\begin{aligned}
& \operatorname{dim} H^{0}\left(C, \mathcal{O}_{C}(m D+\lceil-G\rceil)\right)-\operatorname{dim} H^{1}\left(C, \mathcal{O}_{C}(m D+\lceil-G\rceil)\right) \\
= & \operatorname{deg}(m D+\lceil-G\rceil)-g+1>\operatorname{deg}\left(K_{C}+\{G\}\right)-g+1 \\
\geq & 2 g-2-g+1=g-1 \geq 0
\end{aligned}
$$

for every $m \geq a$, where $g$ denotes the genus of $C$.

Lemma 5.3 (Non-vanishing theorem II). Let $(X, \Delta)$ be a klt surface and let $\pi: X \rightarrow S$ be a proper surjective morphism with $\operatorname{dim} S \geq 1$. Let $D$ be a $\pi$-nef Cartier divisor on $X$ such that $a D-\left(K_{X}+\Delta\right)$ is $\pi$-nef and $\pi$-big for some positive integer $a$. Then there exists a positive integer $m^{\prime}$ such that $\pi_{*} \mathcal{O}_{X}(m D) \neq 0$ for every integer $m \geq m^{\prime}$.

Proof. If $\operatorname{dim} S=2$, then $\pi_{*} \mathcal{O}_{X}(m D) \neq 0$ obviously holds for every integer $m$. Therefore, we may assume that $S$ is a smooth curve with $\pi_{*} \mathcal{O}_{X} \simeq \mathcal{O}_{S}$. We may further assume that every fiber of $\pi: X \rightarrow S$ is irreducible by shrinking $S$ suitably. In this case, $D$ is $\pi$-ample or $\pi$-numerically trivial since $\pi$ is flat. When $D$ is $\pi$-ample, the statement is obvious. 
When $D$ is $\pi$-numerically trivial, $m D-\left(K_{X}+\Delta\right)$ is $\pi$-nef and $\pi$-big for every integer $m$. Therefore, $R^{i} \pi_{*} \mathcal{O}_{X}(m D)=0$ for every integer $m$ and every $i>0$ by Theorem 2.4. This implies that $\pi_{*} \mathcal{O}_{X}(m D) \neq 0$ for every integer $m$ since $\pi$ is flat and $\pi_{*} \mathcal{O}_{X} \neq 0$. Note that

$$
\operatorname{dim} H^{0}\left(F, \mathcal{O}_{F}(m D)\right)=\chi\left(F, \mathcal{O}_{F}(m D)\right)=\chi\left(F, \mathcal{O}_{F}\right)=\operatorname{dim} H^{0}\left(F, \mathcal{O}_{F}\right)>0
$$

holds for every integer $m$, where $F$ is the generic fiber of $\pi: X \rightarrow S$.

Let us explain the proof of Theorem 5.1. We only give a sketch of the proof because the arguments are more or less well known.

Sketch of proof of Theorem 5.1. Here, we will explain how to prove Theorem 5.1. Without loss of generality, we may assume that $S$ is affine and $\pi_{*} \mathcal{O}_{X} \simeq \mathcal{O}_{S}$. By Kodaira's lemma (see [5, Lemma 2.1.29]) and perturbing the coefficients of $\Delta$ slightly, we further assume that $a D-\left(K_{X}+\Delta\right)$ is $\pi$-ample and that $\Delta$ is a $\mathbb{Q}$-divisor.

Case 1. We assume that the characteristic of the base field $k$ is positive and $\operatorname{dim} S=0$.

If $D$ is not numerically trivial, then the statement follows from 10 , Theorem 3.2]. If $D$ is numerically trivial, then $\operatorname{Pic}(X)$ is a free abelian group of finite rank by [8, Theorem 1.5] since $-\left(K_{X}+\Delta\right)$ is ample. Therefore, we have $D \sim 0$. Hence we obtain the desired statement when $\operatorname{dim} S=0$ and the characteristic of $k$ is positive.

Case 2. We assume that $(X, \Delta)$ is klt and the characteristic of the base field $k$ is zero.

This case is a special case of the well-known basepoint-free theorem for klt pairs. It can be proved by the traditional X-method, that is, a clever application of the KawamataViehweg vanishing theorem mainly due to Yujiro Kawamata. For the details of the Xmethod, see [2, Section 4.2].

Case 3. We assume that $(X, \Delta)$ is not klt. We further assume that $\operatorname{dim} S \geq 1$ holds when the characteristic of the base field $k$ is positive.

In this case, a slightly modified version of the X-method works by the vanishing theorem (see Theorem 2.4) and the non-vanishing theorem (see Lemma 5.2). There are no difficulties to adapt the arguments in the proof of [10, Theorem 3.2] for our setting.

Case 4. We assume that the characteristic of the base field $k$ is positive, $\operatorname{dim} S \geq 1$, and $(X, \Delta)$ is klt.

In this case, we first use Lemma 5.3 and apply the traditional $X$-method. Then we can obtain the desired basepoint-freeness by the vanishing theorem (see Theorem 2.4) and the non-vanishing theorem (see Lemma 5.2 .

Therefore, we obtain the desired basepoint-freeness for log surfaces.

As a corollary of Theorem 5.1, we have the following statement. Theorem 5.4 is much sharper than Corollary 4.2 because a GMRLC surface is a log surface by definition. 
Theorem 5.4 (Basepoint-freeness for log surfaces II). Let $(X, \Delta)$ be a log surface and let $\pi: X \rightarrow S$ be a projective morphism onto a variety $S$. Let $D$ be a $\pi$-nef Cartier divisor on $X$ such that $a D-\left(K_{X}+\Delta\right)$ is $\pi$-ample for some positive integer a. Then there exists a positive integer $m_{0}$ such that $\mathcal{O}_{X}(m D)$ is $\pi$-generated for every integer $m \geq m_{0}$.

Proof. Without loss of generality, we may assume that $S$ is affine and $\pi_{*} \mathcal{O}_{X} \simeq \mathcal{O}_{S}$. We take a $\pi$-ample Cartier divisor $H$ on $X$. Then we can take a large positive integer $b$ and an effective Weil divisor $G$ such that $\lfloor\Delta\rfloor+b H \sim G \geq 0$ and that Supp $G$ contains no irreducible components of $\lfloor\Delta\rfloor$. By replacing $\Delta$ with $\Delta-\varepsilon\lfloor\Delta\rfloor+\varepsilon G$, which is $\mathbb{Q}$-linearly equivalent to $\Delta+\varepsilon b H$, for $0<\varepsilon \ll 1$, we may further assume that $\lfloor\Delta\rfloor=0$. Then this theorem follows from Theorem 5.1

For the reader's convenience, we explicitly state the cone and contraction theorem for $\log$ surfaces (see also [11, Theorem 4.4]).

Theorem 5.5 (Cone and contraction theorem for log surfaces). Let $(X, \Delta)$ be a log surface and let $\pi: X \rightarrow S$ be a projective morphism onto a variety $S$. Then the equality

$$
\overline{\mathrm{NE}}(X / S)=\overline{\mathrm{NE}}(X / S)_{K_{X}+\Delta \geq 0}+\sum_{j} R_{j}
$$

and the following hold.

(i) $R_{j}$ is a $\left(K_{X}+\Delta\right)$-negative extremal ray of $\overline{\mathrm{NE}}(X / S)$ for every $j$.

(ii) Let $H$ be a $\pi$-ample $\mathbb{R}$-divisor on $X$. Then there are only finitely many $R_{j}$ 's included in $\left(K_{X}+\Delta+H\right)_{<0}$. In particular, the $R_{j}$ 's are discrete in the half-space $\left(K_{X}+\Delta\right)_{<0}$.

(iii) Let $R$ be a $\left(K_{X}+\Delta\right)$-negative extremal ray of $\overline{\mathrm{NE}}(X / S)$. Then there exists a contraction morphism $\varphi_{R}: X \rightarrow Y$ over $S$ with the following properties.

(a) Let $C$ be an irreducible curve on $X$ such that $\pi(C)$ is a point. Then $\varphi_{R}(C)$ is a point if and only if $[C] \in R$.

(b) $\mathcal{O}_{Y} \simeq\left(\varphi_{R}\right)_{*} \mathcal{O}_{X}$.

(c) Let $\mathcal{L}$ be a line bundle on $X$ such that $\mathcal{L} \cdot C=0$ for every curve $C$ with $[C] \in R$. Then there exists a line bundle $\mathcal{M}$ on $Y$ such that $\mathcal{L} \simeq \varphi_{R}^{*} \mathcal{M}$ holds.

We note that the cone and contraction theorem holds for log surfaces $(X, \Delta)$ without any extra assumptions on $(X, \Delta)$.

Sketch of proof of Theorem 5.5. If the characteristic of the base field $k$ is zero, then Theorem 5.5 follows from [3, Theorem 3.2], which is a special case of [2, Theorem 1.1]. Although [3, Theorem 3.2] (see also [2, Theorem 1.1]) is stated only for the case where $k=\mathbb{C}$, 
it holds true under the assumption that the characteristic of $k$ is zero. This is because the Kodaira-type vanishing theorems in [2] hold true when the characteristic of $k$ is zero by the Lefschetz principle. For the details of the cone and contraction theorem in characteristic zero, we strongly recommend the reader to see [2].

From now on, we assume that the characteristic of $k$ is positive. Then the cone theorem, that is, (i) and (ii), holds true by [9. Theorem 6.2]. Therefore, it is sufficient to prove the contraction theorem (iii). We can take a $\pi$-nef Cartier divisor $D$ on $X$ such that $R=\overline{\mathrm{NE}}(X / S) \cap D^{\perp}$ by (ii). Then $a D-\left(K_{X}+\Delta\right)$ is $\pi$-ample for some positive integer a. By Theorem 5.4, there exists a positive integer $m_{0}$ such that $\mathcal{O}_{X}(m D)$ is $\pi$-generated for every integer $m \geq m_{0}$. We take the Stein factorization of the associated morphism. Then we have a contraction morphism $\varphi_{R}: X \rightarrow Y$ over $S$ satisfying (a) and (b). By construction, $-\left(K_{X}+\Delta\right)$ is $\varphi_{R}$-ample. Therefore, $\mathcal{L}-\left(K_{X}+\Delta\right)$ is also $\varphi_{R}$-ample since $\mathcal{L}$ is relatively numerically trivial over $Y$. By Theorem 5.4 again, we see that there exists a positive integer $n_{0}$ such that $\mathcal{L}^{\otimes n}$ is $\varphi_{R}$-generated for every integer $n \geq n_{0}$. This implies (c).

We note that $R_{j}$ in Theorem 5.5 is spanned by a rational curve.

Theorem 5.6. (Extremal rational curves, see [3, Proposition 3.8]) Let $(X, \Delta)$ be a log surface and let $\pi: X \rightarrow S$ be a projective morphism onto a variety $S$. Let $R$ be a $\left(K_{X}+\Delta\right)$ negative extremal ray of $\overline{\mathrm{NE}}(X / S)$. Then $R$ is spanned by a rational curve $C$ on $X$ such that

$$
0<-\left(K_{X}+\Delta\right) \cdot C \leq 3
$$

Moreover, if $X \not \mathbb{P}^{2}$, then we can choose $C$ with

$$
0<-\left(K_{X}+\Delta\right) \cdot C \leq 2
$$

Proof. By the vanishing theorem in 7, Theorem 6.2], which holds true over any algebraically closed field $k$ of arbitrary characteristic, the proof of [3, Proposition 3.8] works without any changes.

Finally, we give a sketch of the proof of Theorem 1.5, which is the main result of this short paper.

Sketch of proof of Theorem 1.5. Since $(X, \Delta)$ is a log surface that is projective over $S$, we can apply the cone and contraction theorem to $(X, \Delta)$ (see Theorem 5.5). By Lemma 4.3 , we can run the minimal model program under the assumption that $(X, \Delta)$ is GMRLC. After finitely many steps, the minimal model program terminates. By construction, $K_{X}+$ $\Delta$ is pseudo-effective over $S$ if and only if so is $K_{X^{*}}+\Delta^{*}$. By the abundance theorem (see Theorem 4.1], $K_{X^{*}}+\Delta^{*}$ is semi-ample over $S$ when $K_{X^{*}}+\Delta^{*}$ is nef over $S$. 
If $(X, \Delta)$ is $\log$ canonical, then we can easily see that $\left(X_{i}, \Delta_{i}\right)$ is also log canonical for every $i$ by the negativity lemma. Hence (1) is the classical minimal model theory for log canonical surfaces. For the details, see [3, 9] (see also [7]).

The case where $(X, \Delta)$ is a $\mathbb{Q}$-factorial log surface (see $(2))$ was established in characteristic 0 and $p>0$ in [3] and [9], respectively.

If $(X, \Delta)$ is MRLC, then $\left(X_{i}, \Delta_{i}\right)$ is also MRLC by Lemma 4.3. Therefore, (3) holds. Note that (3) is the minimal model program sketched in [1, Section 10].

Theorem 1.5 covers the minimal model theory for log canonical surfaces, $\mathbb{Q}$-factorial log surfaces, and MR log canonical surfaces.

We note that some results in this short paper were already discussed in a more general setting by Hiromu Tanaka. We recommend the interested reader to see [11]. We also note that the minimal model theory established in [3] was generalized for log surfaces in Fujiki's class $\mathcal{C}$ in 6$]$.

\section{Acknowledgments}

The author was partially supported by JSPS KAKENHI Grant Numbers JP16H03925, JP16H06337. He thanks Kento Fujita, Kenta Hashizume, Haidong Liu, and Hiromu Tanaka for useful comments. He also thanks Keisuke Miyamoto for pointing out some mistakes. Finally, he thanks the referee for some useful comments and suggestions.

\section{References}

[1] V. Alexeev, Boundedness and $K^{2}$ for $\log$ surfaces, Internat. J. Math. 5 (1994), no. 6, 779-810.

[2] O. Fujino, Fundamental theorems for the log minimal model program, Publ. Res. Inst. Math. Sci. 47 (2011), no. 3, 727-789.

[3] _ Minimal model theory for log surfaces, Publ. Res. Inst. Math. Sci. 48 (2012), no. $2,339-371$.

[4] _ On log canonical rational singularities, Proc. Japan Acad. Ser. A Math. Sci. 92 (2016), no. 1, 13-18.

[5] _ Foundations of the Minimal Model Program, MSJ Memoirs 35, Mathematical Society of Japan, Tokyo, 2017.

[6] Minimal model theory for log surfaces in Fujiki's class $\mathcal{C}$, to appear in Nagoya Math. J. 
[7] O. Fujino and H. Tanaka, On log surfaces, Proc. Japan Acad. Ser. A Math. Sci. 88 (2012), no. 8, 109-114.

[8] R. Ohta and S. Okawa, On ruled surfaces with big anti-canonical divisor and numerically trivial divisors on weak log Fano surfaces, to appear in Manuscripta Math.

[9] H. Tanaka, Minimal models and abundance for positive characteristic log surfaces, Nagoya Math. J. 216 (2014), 1-70.

[10] The X-method for klt surfaces in positive characteristic, J. Algebraic Geom. 24 (2015), no. 4, 605-628.

[11] _ Minimal model program for excellent surfaces, Ann. Inst. Fourier (Grenoble) 68 (2018), no. 1, 345-376.

Osamu Fujino

Department of Mathematics, Graduate School of Science, Osaka University, Toyonaka, Osaka 560-0043, Japan

E-mail address: fujino@math.sci.osaka-u.ac.jp 